\title{
Construcción de un cuestionario para la Valoración de los Efectos Subjetivos de Sustancias con Potencial de Abuso (VESSPA): Evaluación del éxtasis'
}

\author{
Sandra Poudevida Jürschik*, Magí farré Albaladejo**, Pere N. Roset Arissó***, Jordi Camí Morell**** \\ *Doctora, Psicóloga Clínica. Unidad de Farmacología, Instituto Municipal de Investigación Médica (IMIM). \\ **Doctor, Farmacólogo Clínico. Consultor 2 de la Unidad de Farmacología del Instituto Municipal de Investigación Médica (IMIM). \\ Profesor titular de Farmacología de la Universidad Autónoma de Barcelona. \\ ***Doctor, Farmacólogo Clínico. Profesor asociado de Farmacología de la Universidad Autónoma de Barcelona. \\ ****Doctor, Farmacólogo Clínico. Director del Instituto Municipal de Investigación Médica (IMIM) \\ Catedrático de Farmacología de la Universidad Pompeu Fabra.
}

\section{RESUMEN}

Se construye y valida un instrumento para medir los efectos subjetivos producidos por la 3,4-metilenodioximentanfetamina (MDMA). Tras la construcción de un banco de ítems, posteriores reducciones y un análisis factorial, se obtuvo un cuestionario de 36 ítems al que se le denominó VESSPA (Valoración de Efectos Subjetivos de Sustancia con Potencial de Abuso). El cuestionario consta de 6 escalas: "Sedación", "Somatización Ansiosa," "Cambios de Percepción", "Placer y Contacto Social", "Actividad y Energía" y escala de "Sintomatología Psicótica". Respecto a la fiabilidad se obtuvo una consistencia interna entre 0.67 y 0.86 , así como un índice de correlación del testretest entre 0.79 y 0.91 , según la escala. En cuanto a la validez se aplicaron tres pruebas: correlaciones entre escalas del VESSPA y del ARCI (Addiction Research Center Inventory-49 item short form), puntuación de las diferentes escalas frente a otras condiciones simuladas (alcohol, cannabis, cocaína y LSD) y respuesta del cuestionario en situación experimental en un ensayo clínico en el que se administró MDMA y alcohol. Los resultados demuestran que el cuestionario VESSPA es un instrumento válido y fiable para medir los efectos farmacológicos de la MDMA y de otros psicofármacos, y puede ser utilizado también en ensayos clínicos.

Palabras clave: éxtasis, MDMA, cuestionario, validación, efectos subjetivos, VESSPA.

\section{ABSTRACT}

The objective of this study was to construct an instrument capable of measuring the subjective effects produced by 3.4methyilenedioxymethamphetamine (MDMA). After the construction of a bank of items, subsequent reductions and a factorial analysis, a 36-item questionnaire was obtained with a five-point Likert response. This questionnaire was called VESSPA-SEE (Evaluation of Subjective Effects of Substances with Abuse Potential) and contains 6 scales: Sedation, Physical Anxiety, Changes in Perception, Pleasure and Social Contact, Activity and Energy, and Psychotic Symptoms. For these scales, the coefficients of alpha ranged from 0.67 to 0.86 , and the coefficients of test-retest reliability ranged from 0.79 to 0.91 . The validity of the scale was investigated by the application of three tests: correlations with VESSPA and ARCI scales (Addiction Research Center Inventory-49 item short form), scores of the different scales in other simulated conditions (alcohol, cannabis, cocaine and LSD), and response of the questionnaire in an experimental situation in a clinical trial in where MDMA and alcohol were administered. The results showed that the VESSPA questionnaire is an instrument with an acceptable validity and reliability for measuring the pharmacological effects of the MDMA and other psychopharmacos. In addition, the questionnaire seems apropiate for use in a clinical trial context.

Key words: ecstasy, MDMA, questionnaire, validation, subjective effects, VESSPA.

\section{INTRODUCCIÓN}

a 3,4-metilenodioximentanfetamina (MDMA), popularmente conocida con el nombre de éxtasis o XTC, ha despertado interés durante la últi- ma década por su elevado uso entre los jóvenes y su asociación a movimientos festivos y de ocio. En la mayoría de países parece que su consumo está aún en expansión. El Ministerio del Interior lleva a cabo desde hace unos años una encuesta poblacional y una

(1) Este trabajo ha obtenido el Premio Zambón-Socidrogalcohol 2003. 
encuesta escolar sobre drogas que recoge en una serie de informes bianuales (Delegación del Gobierno para el Plan Nacional sobre Drogas, 1995-2002). En estos informes puede observarse el aumento del consumo de éxtasis en el año 2000, así como la disminución de la edad de inicio del consumidor y la creciente presencia femenina. No obstante se observa un mayor grado de sensibilización sobre los riesgos o problemas que lleva asociado el consumo de estas sustancias. Los resultados del último informe sobre la encuesta domiciliaria (Delegación del Gobierno para el Plan Nacional sobre Drogas, 2001) apuntan a que la MDMA fue consumida alguna vez por el $2.4 \%$ de los encuestados (4.7\% en la población de 15-29 años). En el último informe de la encuesta del consumo escolar de la Delegación del Gobierno para el Plan Nacional sobre Drogas (1996-2002) se entrevistó a 20.450 estudiantes de 14 a 18 años, de enseñanza secundaria y formación profesional. El porcentaje de población escolar que ha tomado éxtasis es del $5.7 \%$ y un $4.6 \%$ lo hizo durante el último año, ambas cifras preocupantes por incrementarse en un $2 \%$ sobre el informe anterior. Algo similar ha ocurrido a nivel europeo. En un informe del Observatorio Europeo de las Drogas y las Toxicomanías (2000) sobre el problema de la drogodependencia en la Unión Europea, se presenta una comparación de la prevalencia del consumo de éxtasis en diferentes países. Anfetaminas y éxtasis son el segundo tipo de droga más consumido en la UE. Las drogas de diseño las ha tomado alguna vez entre el $1 \%$ y el $5 \%$ de los jóvenes (de entre 16 y 34 años) aunque en algunos grupos el consumo llega hasta el $10 \%$ según el país. Los datos referidos al consumo en algunos países, entre la población de 15 a 34 años son los siguientes: Reino Unido $(10 \%$ de la población de entre 16 y 29 años), España (4.7\%), Holanda (4.4\%), Alemania (3.2\%), Bélgica (1.3\%), Suiza (1\%) y Grecia $(0.6 \%)$. Estos datos hacen que exista cierta alarma social y sanitaria por sus efectos agudos, pudiendo causar intoxicaciones mortales, y por las posibles consecuencias a largo plazo, asociadas a su conocida neurotoxicidad en animales.

La MDMA produce algunos de los efectos típicos de los psicoestimulantes (euforia, bienestar, energia, alerta), además de incrementar la empatía y sociabilidad y producir alteraciones en las percepciones que van desde incrementos leves de agudeza visual y auditiva hasta llegar incluso a las ilusiones y alucinaciones (estas últimas en casos muy puntuales de consumo y acompañadas de un contexto lúdico-festivo). Por tanto se ha considerado como una sustancia que podría clasificarse entre los estimulantes y los alucinógenos (de la Fuente y cols., 1997). Además posee una serie de características empatógenas o entactógenas referentes al deseo de contacto con los demás y de sociabilidad (Camí y Farré, 1996; Solowij y cols., 1992; Liester y cols., 1992) que no han sido evaluadas hasta el momento. En general, sus efectos subjetivos se han estudiado principalmente por encuestas a consumidores en condiciones no experimentales, donde el sujeto recuerda lo que sintió bajo los efectos de la sustancia (Peroutka y cols., 1988; Solowij y cols., 1992; Liester y cols., 1992; Davidson y Parrott, 1997; Williamson y cols., 1997, Curran y Travill, 1997). Una limitación de esta aproximación es que no se conoce lo que realmente se consume, no se sabe la dosis, la influencia del setting, las combinaciones, etc. De hecho, cuando intentamos medir fenómenos de cualquier tipo, la gran ambición es el control de todas las variables contaminantes con la intención de atribuir el cambio a aquello que es nuestro objeto de estudio. Para conocer de forma más precisa los efectos subjetivos de los fármacos psicoactivos se debe recurrir a los estudios experimentales en los que se administra la sustancia en condiciones controladas recogiendo de forma narrativa los efectos o bien recogiendo los efectos mediante cuestionarios (método que permite establecer comparaciones posteriores con otros estudios y sustancias). Para evitar las influencias de las expectativas estos estudios se realizan de forma enmascarada o ciega, con algún tipo de sustancia control, por ejemplo placebo y con asignación aleatoria de los sujetos a las diferentes condiciones. Por el contrario, una desventaja de estos estudios es su falta de naturalidad al crearse una situación artificial de consumo.

Por lo tanto, en un ensayo clínico (investigación realizada en condiciones experimentales controladas) se administra la sustancia al sujeto y se miden los efectos tanto fisiológicos como psicológicos. Si se quiere estudiar el curso temporal de los efectos se requiere el uso de cuestionarios estructurados que puedan administrarse de forma repetida para seguir el transcurso de los mismos. Una limitación para el estudio de la MDMA es que los cuestionarios existentes sólo evalúan los efectos estimulantes o los alucinógenos por separado y no son capaces de profundizar en las propiedades empatógenas ni cubrir los cambios leves en las percepciones, que por otra parte parecen ser una de las características diferenciales de esta sustancia. Al no haber apenas estudios controlados sobre la sustancia, no se había presentado la necesidad de medir sus efectos subjetivos y además no existen cuestionarios capaces de medirlos en un sólo instrumento.

Los primeros estudios de los efectos subjetivos del la MDMA se realizaron mediante listas de adjetivos "hechos a medida", es decir, se efectuaba un listado de efectos mediante preguntas a consumidores, y este listado era administrado a los sujetos voluntarios definitivos del estudio. Estos listados no pasaban ningún tipo de prueba de fiabilidad o validez, y además, al ser construidos para cada estudio en particular, resultaba difícil comparar los resultados entre unos estu- 
dios y otros. Numerosos autores (Greer y Tolbert, 1986; Dowling y cols., 1987; Peroutka y cols., 1988; Liester y cols., 1992; Henry y cols., 1993; Grob y cols., 1996) se han servido de esta técnica, haciendo buenas descripciones de los efectos subjetivos del éxtasis, pero de una forma poco reproducible. Como veremos más adelante, estas listas de efectos servirán para la construcción de nuestro cuestionario ya que su finalidad fue la descripción de los efectos característicos del éxtasis como euforia, energía, sensualidad, felicidad, deseo de contacto, etc.

El objetivo de este estudio fue construir un cuestionario capaz de medir el amplio espectro de efectos subjetivos producidos por el éxtasis. Este cuestionario podría ser sensible también a los efectos de otras drogas de abuso debido a la no exclusividad de los mismos a una droga en concreto (de hecho sería de esperar que algunas escalas fueran más sensibles a otras drogas). La medición de los efectos subjetivos es importante a la hora de su conocimiento ya que permite comparar diferentes sustancias, a distintas dosis, y diferentes estudios. Su utilidad se evaluó en condiciones experimentales y simuladas y los resultados se compararon con el cuestionario $\mathrm{ARCl}$ que puede medir alguno de los efectos propuestos.

\section{SUJETOS, MATERIALY PROCEDIMIENTO}

\section{Sujetos}

En total participaron en el estudio 265 consumidores de éxtasis. De éstos, 40 sujetos $(31$ hombres y 9 mujeres) participaron en las entrevistas exploratorias realizadas para la segunda reducción de ítems. A continuación se administró un precuestionario de 85 ítems a 195 consumidores de éxtasis de los cuales 102 contestaron a esta condición (64 hombres y 38 mujeres) y 93 a otras condiciones (cocaína, cannabis, LSD o alcohol) (57 hombres y 36 mujeres). Por último, 30 sujetos (15 hombres y 15 mujeres) participaron en las pruebas de fiabilidad de test-retest y 9 varones en un ensayo clínico en el que se administró MDMA como sustancia principal.

\section{Tabla 1. Consistencia interna de las escalas del VESSPA}

\begin{tabular}{|ll|}
\hline Escala & Alfa \\
\hline SEDACIÓN & 0.75 \\
\hline SOMATIZACIÓN ANSIOSA & 0.82 \\
\hline CAMBIOS DE PERCEPCIÓN & 0.83 \\
\hline PLACERY CONTACTO SOCIAL & 0.86 \\
\hline ENERGÍAY ACTIVIDAD & 0.86 \\
\hline SINTOMATOLOGÍA PSICÓTICA & 0.67 \\
\hline
\end{tabular}

El presente proyecto de investigación fue aprobado por el Comité Ético Local (CEIC-IMAS 98/801). Los sujetos firmaron previamente un consentimiento informado que cumplía todas las normas éticas de privacidad y confidencialidad requeridas en la Declaración de Helsinki (World Medical Association, 1996). Participaron voluntarios de ambos sexos mayores de 18 años. La participación fue libre y voluntaria, el sujeto podía abandonar en cualquier momento la investigación. Por el tiempo perdido y gastos de desplazamiento se les compensaba con una pequeña donación económica.

\section{Material y procedimiento}

En primer lugar se construyó un banco de ítems partiendo de cuestionarios ya validados para otras sustancias que recogían efectos que el éxtasis también puede producir como los de tipo estimulante, alucinógeno y sedante (estos últimos sólo a dosis altas). Estos cuestionarios fueron:

1) El Addiction Research Center Inventory-49 items short form (ARCI) por ser uno de los cuestionarios más utilizados en el campo de los ensayos clínicos de sustancias con potencial de abuso. El ARCl de 49 ítems es un cuestionario de respuesta verdadero o falso, traducido y validado por Lamas y cols. (1994). Contiene cinco escalas que miden sedación (escala PCAG: Pentobarbital-Clorpromazina-Alcohol), euforia (escala MBG: grupo Morfina-Benzedrina), disforia o efectos desagradables (escala LSD: Dietilamida del Acido Lisérgico), eficiencia intelectual (escala BG: grupo Benzedrina) y efectos de tipo anfetamínico (escala A: Anfetamina). Un estudio reciente realizado en nuestro centro, demuestra su sensibilidad a sustancias sedantes, estimulantes y opioides en el transcurso de ensayos clínicos con dichas sustancias (Arasteh y cols., 1999). También algunas escalas del $\mathrm{ARCl}$ han mostrado ser sensibles en varios ensayos clínicos con MDMA para evaluar efectos euforizantes y de tipo anfetamínico (Camí y cols., 2000). Sin embargo, el ARCI no evalúa efectos perceptivos o alucinaciones ni empatía o sociabilidad. Aunque este era el cuestionario que más se aproximaba a los objetivos

\begin{tabular}{|ll|}
\hline \multicolumn{2}{|c|}{ Tabla 2. Correlación test-retest } \\
Escala & Correlación \\
\hline SEDACIÓN & 0.91 \\
\hline SOMATIZACIÓN ANSIOSA & 0.81 \\
\hline CAMBIOS DE PERCEPCIÓN & 0.79 \\
\hline PLACERY CONTACTO SOCIAL & 0.81 \\
\hline ENERGÍAY ACTIVIDAD & 0.85 \\
\hline SINTOMATOLOGÍA PSICÓTICA & 0.83 \\
\hline
\end{tabular}


de la presente investigación, por su actual utilización y diversidad de escalas, se desestimó por carecer de una escala específica para la medición de efectos característicos de la MDMA. No por ello se desestimaron sus ítems y formaron parte del primer listado de ítems del nuevo cuestionario.

2) También se tuvieron en cuenta los ítems de la Hallucinogen Rating Scale (HRS), un cuestionario especialmente diseñado para medir los efectos de drogas alucinógenas como la N, N-dimetiltriptamina (DMT) y la ayahuasca y que ha sido utilizado en ensayos clínicos con dichas drogas (Strassman y cols., 1994; Riba y cols., 2001a; 2001b). Consta de 100 ítems cuya respuesta es tipo Likert, con 5 grados de respuesta y contiene 6 escalas que miden: afecto, somastesia, percepción, cognición, volición e intensidad.

3) Por último se tomaron los ítems de las Escalas Analógicas Visuales (EAV) diseñadas para ensayos clínicos en los que se administró MDMA y se adaptaron sus adjetivos a los efectos de la sustancia tras consultas con expertos (Camí y cols., 2000). Algunas de ellos son: "estimulado", "colocado", "malos efectos", "le gusta el fármaco", "borracho", "somnolencia", "activo", "pasivo", "concentrado" y "rendimiento".

Para completar el listado, y puesto que estos cuestionarios no contemplaban efectos concretos del éxtasis de tipo entactógeno o de sociabilidad, también se realizó un repaso a la literatura científica sobre los efectos del éxtasis y se tomaron descripciones de los efectos obtenidos a través de encuestas o bien listados realizados por expertos en el tema (Greer y Tolbert, 1986; Dowling y cols., 1987; Peroutka y cols., 1988; Liester y cols., 1992; Solowij y cols., 1992; Henry y cols., 1993; Camí y Farré., 1996; Grob y cols., 1996; Burnat y cols., 1996).

Finalmente se obtuvo un listado de 267 ítems y sobre este listado se efectuaron dos reducciones del listado, la primera mediante consultas a expertos y la segunda mediante entrevistas a 40 consumidores de éxtasis, tomando aquellos ítems en que los voluntarios contestaron afirmativamente en más de un 50\% para los efectos producidos por el éxtasis (ver Poudevida y cols., 1997 para más información).

Tras estas reducciones se obtuvo un precuestionario de 85 ítems al que se aplicó una respuesta tipo Likert de 0 (ausencia de efecto) a 4 (efecto máximo) para contemplar la intensidad del efecto. Este precuestionario recogía efectos de tipo entactógeno, estimulante, alucinógeno y sedante. Este precuestionario de 85 ítems se administró a 195 sujetos. Todos ellos contestaron bajo condiciones simuladas de estar bajo los efectos de tres drogas, ya que se trataba de policonsumidores en su totalidad. Bajo la condición simulada de éxtasis contestaron al precuestionario 102 sujetos, 51 sujetos lo hicieron para la condición simu- lada de cocaína, 61 para el cannabis, 50 para la condición LSD y 38 para el alcohol (algunos sujetos contestaron a más de una condición).

\section{Resultados}

La reducción final del cuestionario se realizó basándose en la administración del mismo a los 102 sujetos que habían contestado bajo la condición de éxtasis, mientras las otras condiciones sirvieron para la validación del cuestionario, pero no para la fase de reducción (ver más adelante). Partiendo del banco de 85 ítems, en primer lugar se realizó un análisis factorial exploratorio usando el método de componentes principales con rotación varimax. Se obtuvieron 15 factores con un valor propio mayor que 1 que explicaban un $69.0 \%$ de la varianza. Se observó que en los cuatro primeros factores que tenían un valor propio mayor que 3 y agrupaban 63 ítems que explicaban el 48.8\% de la varianza. En el primer factor se agrupaban 26 ítems, 18 en el segundo, 11 en el tercero y 8 ítems en el cuarto. A partir del quinto factor se agrupaban tan sólo 3 o 4 ítems en cada uno, cifra que parecía demasiado reducida a la hora de formar una escala. Se realizó a continuación un análisis factorial forzando el número de factores a cuatro pensando que este sería el número correcto de categorías o escalas. Se observó que el primer factor reunía ítems claramente diferenciados en tres categorías que hacían referencia a sintomatologías de carácter distintas: sedación, somatización ansiosa y cambios de percepción. El segundo factor reunía síntomas referentes a sensaciones placenteras y sociales. Los ítems del tercer factor aludían a una sintomatología que reflejaba actividad y energía. El cuarto factor se caracterizaba por describir sintomatología de tipo psicótica. Para la selección de los ítems definitivos se convocaron diferentes reuniones de expertos en las que se propuso la discusión de un análisis cualitativo de los datos obtenidos. Se utilizó como base esta reducción a 4 factores, aunque conceptual y cualitativamente se formaron 6 escalas (tres que se derivaban del primer factor y otras tres del segundo, tercer y cuarto factor respectivamente). Se tuvo en consideración el peso factorial que presentaba el ítem en el factor (> de 0.4).

Por último, se optó por un cuestionario de seis escalas formadas por seis ítems cada una, lo que en principio pareció un número razonable para medir los efectos del éxtasis y otras sustancias así como suficientemente corto para poder ser administrado repetidamente a lo largo de un ensayo clínico. Para verificar la bondad de las escalas se realizó un nuevo análisis factorial para confirmar previsiones con el nuevo cuestionario de 36 ítems forzando el número de factores a 6 , dado que se preveían 6 escalas para el mismo. Se observó que los ítems se agrupaban de la manera esperada. 
Tabla 3. Coeficientes de correlación de Pearson entre las escalas del VESSPA y del ARCI

\begin{tabular}{lllllll} 
Escala & PCAG & MBG & LSD & BG & A \\
\hline Sedación & $0.62^{* *}$ & -0.08 & $0.31^{* *}$ & $-0.36^{*}$ & 0.07 \\
\hline Somatización ansiosa & $0.32^{* *}$ & 0.09 & $0.38^{* *}$ & -0.15 & 0.04 \\
\hline Cambios de percepción & $0.32^{* *}$ & 0.06 & 0.14 & $-0.37^{*}$ & -0.06 \\
\hline Placer y contacto social & -0.15 & $0.57^{* *}$ & -0.08 & 0.18 & 0.17 \\
\hline Actividad y energía & $-0.36^{* *}$ & $0.22^{*}$ & -0.02 & $0.31^{*}$ & 0.15 \\
\hline Sintomatología psicótica & $0.31^{* *}$ & -0.04 & $0.21^{*}$ & -0.10 & 0.06 \\
\hline
\end{tabular}

PCAG: Sedación; MBG: Euforia; LSD: Efectos físicos desagradables; BG: Eficiencia intelectual; A: Efectos anfetamínicos. ** La correlación es significativa al nivel $0.01(p<0.01)$. ${ }^{*}$ La correlación es significativa al nivel $0.05(p<0.05)$.

Finalmente se obtuvo un cuestionario al que se denominó VESSPA (Valoración de Efectos Subjetivos de Sustancias con Potencial de Abuso). Este cuestionario, autoadministrado, constaba de 36 ítems en total, dividido en seis escalas de seis ítems cada una. Estas escalas fueron: escala de Sedación, escala de Somatización Ansiosa, escala de Cambios de Percepción, escala de Placer y Contacto Social, escala de Actividad y Energía y escala de Sintomatología Psicótica. Se estableció una respuesta tipo Likert según la intensidad de los efectos $(0=$ nada, $1=$ un poco, 2 =moderado, $3=$ bastante, $4=$ mucho).

\section{Fiabilidad y validez}

Para estimar la fiabilidad del instrumento se calculó la consistencia interna de cada una de las escalas mediante el alfa de Cronbach obteniendo puntuaciones entre 0.67 y 0.86 , consistencia que mostró ser aceptable formando escalas homogéneas ya que sus ítems estaban bien relacionados entre sí (tabla 1). Para informar sobre la constancia temporal del instrumento de evaluación, se administró el cuestionario a 30 sujetos ( $50 \%$ varones; edad media=23.2 años; intervalo $=18-31$ ) en dos ocasiones separadas por un intervalo de tiempo mínimo de 1 semana (media=;11.1 días; d.s. = 5.3 días). Los índices obtenidos entre el test y el retest oscilaron entre 0.79 y 0.91 según las escalas. Estas puntuaciones mostraron que el instrumento tenía estabilidad en el tiempo (tabla 2).

En cuanto a las pruebas de validez, se buscaron correlaciones de las escalas del VESSPA con el ARCl49 item short form (tabla 3). Aunque no miden exactamente lo mismo, se podían establecer algunas relaciones lógicas entre sus escalas. La escala de Sedación del VESSPA mostró una alta correlación positiva con la escala PCAG del ARCl $(r=0.62, p<0.01)$ que también mide sedación. La escala de Somatización Ansiosa correlacionó con la escala LSD del ARCI $(r=0.38, p<0.01)$ que mide efectos físicos desagradables de tipo ansioso. La escala de Placer y Contacto Social correlacionó con la escala MBG del $\mathrm{ARCl}$ que mide euforia o sensación de sentirse bien $(r=0.57$, $\mathrm{p}<0.01)$. La escala de Actividad y Energía correlacionó con las escala $B G$ del $A R C l$ que mide eficiencia intelectual $(r=0.31, p<0.05)$ y negativamente con la PCAG (sedación) $(r=-0.36, p<0.05)$.

Como se ha comentado también se administró el VESSPA bajo otras condiciones simuladas aparte del éxtasis. Algunas sustancias podían, y de hecho era de esperar, obtener puntuaciones más elevadas que el éxtasis en algunas escalas ya que aunque el éxtasis puede producir todo este tipo de efectos, otras sustancias también pueden producirlos con mayor intensidad. Concretamente sustancias psicoestimulantes como la cocaína producen efectos estimulantes, sustancias alucinógenas como la LSD producen efectos de tipo alucinógeno y sustancias sedantes como el cannabis o el alcohol producen efectos sedantes. De este modo la cocaína fue la sustancia que más puntuó en las escala de Somatización Ansiosa y la de Actividad y Energía ya que se trata de la droga estimulante por excelencia. El cannabis obtuvo puntuaciones más altas en la escala de Sedación, así como el alcohol, resultados también esperados por tratarse de sustancias claramente sedantes. La LSD obtuvo puntuaciones elevadas en las escalas de Cambios de Percepción y de Sintomatología Psicótica, resultado lógico al tratarse de un alucinógeno clásico. Por último la escala de Placer y Contacto Social, que sería la más adecuada para medir los efectos de tipo entactógeno, hasta el momento no medidos por otros cuestionarios, presentó las puntuaciones más altas para el éxtasis (tabla 4).

Para finalizar, y debido a que el cuestionario VESSPA va a ser utilizado en principio en el contexto de ensayos clínicos, se administró a lo largo de un ensayo clínico en el que se administraron MDMA y alcohol, ya que ambas sustancias se combinan con frecuencia en situación de ocio. En este ensayo clínico participaron 9 sujetos varones sanos. Las condiciones puras del estudio fueron: 100mg de MDMA, 0.8mg/ $\mathrm{Kg}$ de alcohol y placebo. El ensayo clínico fue a doble ciego, cruzado, controlado con placebo y con asigna- 
ción aleatoria de las condiciones de tratamiento (los detalles se encuentran publicados, Hernández-López y cols., 2002). Los resultados obtenidos demostraron incrementos de las puntuaciones de las escalas de Somatización Ansiosa, Placer y Contacto Social, y la de Actividad y Energía cuando se administró MDMA respecto a placebo. La escala de Sedación obtuvo puntuaciones significativamente altas para la condición de alcohol y no se observaron aumentos significativos para las escalas de Cambios de Percepción ni de Sintomatología Psicótica. Estos últimos posiblemente pueden aparecer tras la administración de una mayor dosis de MDMA o en caso de intoxicación (tabla 5).

\section{Discusión}

Los resultados obtenidos en este estudio avalan que el cuestionario VESSPA puede ser un instrumento de utilidad para la medida de los efectos subjetivos del éxtasis. Como se ha comentado, para medir los efectos subjetivos del éxtasis, se utilizaban listas de efectos no validadas o la mera descripción de los mismos, ya que no existía ningún instrumento fiable y válido para la medición de los mismos. Una de las propiedades del VESSPA es que también permite medir los efectos subjetivos de otras sustancias de tipo estimulante, alucinógeno y sedante. La inclusión en este cuestionario de estos efectos subjetivos característicos de otras sustancias bien conocidas ha permitido compararlos con los del éxtasis y analizar las diferencias existentes entre las mismas.

\section{Fiabilidad}

\section{Consistencia interna}

Si observamos los valores de fiabilidad alfa de las escalas, vemos que están entre el 0.67 el más bajo, y 0.86 el más alto. Se trata de una fiabilidad aceptable ya que los valores encontrados en este estudio son usuales en escalas con pocos ítems (Ferrando y Pérez, 1996; Pérez y Torrubia, 1986). Por norma se aceptan valores desde 0.7 a 0.8 hasta 1 , sin embargo la fiabilidad puede verse afectada por un número bajo de ítems. Al revisar la fiabilidad en bloques de 6 ítems se obtienen coeficientes límite sistemáticamente inferiores a los de módulos con mucha más extensión (Renom y cols., 1995). Valores muy similares han sido obtenidos en la validación de otros cuestionarios (Smith, 1999; Tange, 1999; Holzemer y cols. 1999).

\section{Test-retest}

Los resultados en cuanto a la estabilidad en el tiempo de las puntuaciones en las escalas son altamente satisfactorios, siendo de 0.79 el más bajo (escala de Cambios de Percepción) y de 0.91 el más alto (escala de Sedación).

En la validación del $\mathrm{ARCl}$ original, los coeficientes oscilaron alrededor del 0.60 (Haertzen, 1966), coeficiente aceptable, pero inferior al obtenido en el VESSPA. Un coeficiente test-retest es mejor cuanto más se acerque a 1, por consiguiente, coeficientes alrededor del 0.8-0.9 pueden considerarse como buenos (Adams, 1975; Anastasi, 1982; Nunnally y Bernstein, 1994).

\section{Validez}

Correlación entre las escalas del VESSPA y las del $\mathrm{ARCl}$

La combinación de varias pruebas proporciona una validez de constructo y generalización científica mayores en el dominio (como un todo en relación con una medida única). De todas maneras, es difícil encontrar pruebas ya reconocidas y estandarizadas en las que depositar la confianza para comparar sus resultados con el nuevo test (Renom y cols., 1995). Se escogió el $\mathrm{ARCl}$ como medida de referencia debido a que acumula un largo proceso de validación y es uno de los instrumentos más utilizado en el campo de la investigación en toxicomanías aparte de la experiencia propia que existe en nuestro centro con este instrumento. La comparación de estos dos instrumentos no es sencilla, ya que no miden exactamente lo mismo, sin embargo, la relación encontrada entre algunas escalas de estos dos instrumentos podrían apuntar a la validez deI VESSPA.

Tabla 4. Puntuaciones de las diferentes condiciones en cada escala del VESSPA (0-4 puntos)

\begin{tabular}{|lcccccc|} 
& Sedación & Somatización ansiosa & Cambios de percepción & Placer y contacto social & Actividad y energía & Sintomatología psicótica \\
\hline Éxtasis & 0.72 & 2.25 & 1.12 & 2.87 & 2.75 & 0.99 \\
\hline Alcohol & 1.20 & 0.43 & 0.45 & 1.96 & 1.19 & 0.37 \\
\hline Cocaína & 0.20 & 2.56 & 0.37 & 2.25 & 3.12 & 1.02 \\
\hline Cannabis & 1.82 & 0.73 & 0.47 & 1.39 & 0.28 & 0.81 \\
\hline LSD & 0.74 & 1.42 & 2.26 & 2.09 & 2.13 & 1.83 \\
\end{tabular}


La escala de Sedación del VESSPA obtuvo, como era de esperar, una correlación alta y positiva con la escala PCAG (sedación) del ARCI. La PCAG es una escala bien validada en numerosos estudios con sustancias sedantes (Terán y cols., 1993; Farré y cols., 1996; 1997; Holdstock y de Wit, 1998; 1999). También la escala de Sedación del VESSPA reveló una correlación negativa con la escala $\mathrm{BG}$ del $\mathrm{ARCl}$, así como la PCAG vs. la BG en numerosos estudios (Martin y cols., 1971; Fischman y cols., 1976; Nemeth-Coslett y cols., 1986; Cone y cols., 1988a; Chait y cols., 1988; 1993; Johanson, 1991; Preston y cols., 1992).

La escala de Somatización Ansiosa del VESSPA correlacionó positivamente con las escalas LSD, resultado esperable ya que una somatización ansiosa es percibida como un efecto desagradable, efectos que contempla la escala LSD, y que además contempla ítems que reflejan ansiedad o efectos físicos desagradables como "me siento ansioso e irritable", "noto que me tiembla la mano cuando intento escribir" o "noto una creciente percepción de sensaciones corporales".

La escala de Cambios de Percepción registró una correlación positiva con la escala PCAG del ARCI. El éxtasis a dosis altas puede producir distorsiones visuales y es también a dosis altas que suele presentar sedación (Camí y cols., 2000).

La escala de Placer y Contacto Social refleja efectos placenteros de sentirse bien con uno mismo y con los demás. Este concepto está muy cerca de lo que denominamos euforia, definido en el $\mathrm{ARCl}$ precisamente como sentirse bien, y reflejado en su escala MBG. La correlación entre ambas escalas, como era de esperar, fue altamente positiva. La MBG aumenta con sustancias estimulantes como la cocaína (Fischman y cols., 1976; Preston y cols., 1992), y también con sustancias opioides cuyo potencial de abuso está más que demostrado (Jasinski y cols., 1986). Debería administrarse el VESSPA bajo estas condiciones en estudios experimentales para corroborar su sensibilidad a la medición del potencial de abuso de una sustancia.

La escala de Actividad y Energía del VESSPA contiene ítems que reflejan efectos subjetivos de estimulación como la escala $\mathrm{BG}$ del $\mathrm{ARCl}$ (eficiencia intelectual). La BG también aumenta tras la administración de diversos derivados anfetamínicos (Chait y cols., 1986; Martin y cols., 1971) y de cocaína (Fischman y cols., 1976; Preston y cols., 1992). Este resultado también está de acuerdo con la correlación negativa mostrada con la escala PCAG, observado ya en diversos estudios tras la administración de diferentes estimulantes (Fischman y cols., 1976; Preston y cols., 1992; Chait y cols., 1986; Martin y cols., 1971).

La escala de Sintomatología Psicótica presenta correlaciones similares a la escala de Sedación y a la de Somatización Ansiosa. Podríamos englobar los efectos de estas tres escalas dentro de un compendio de efectos "negativos" en tanto que representan los efectos adversos del éxtasis (efectos no buscados por el sujeto). Desplazando este razonamiento al ARCl, tras la administración del éxtasis las escalas con efectos "negativos" percibidos por el sujeto serían la PCAG y la LSD (por lo menos hablando en situación de ocio). Esto podría explicar que la escala de Sintomatología Psicótica correlacione con ambas escalas de efectos "negativos" del ARCI: PCAG y LSD.

\section{Administración del VESSPA bajo otras condiciones (sustancias)}

Era de esperar que unas escalas fueran más sensibles a otras ante ciertas condiciones ya que, como ya se ha comentado, algunas escalas del VESSPA son características de otras sustancias, a pesar de que puedan describir efectos subjetivos también del éxtasis.

La escala de Sedación obtuvo un mayor aumento para las condiciones de cannabis y alcohol, sustancias sedantes por naturaleza. El cannabis es una sustancia sedante que a menudo los sujetos la utilizan para conciliar el sueño o tranquilizarse ante ciertas situaciones que les puede parecer estresantes (trabajo, exámenes, etc.) y por lo tanto, no sólo es un sedante, si no que también lo utilizan como ansiolítico. Numerosos estudios experimentales demuestran el aumento de la escala PCAG del ARCI tras la administración de cannabis (Higgins y Stitzer, 1986; Cone y Johnson, 1988b). El alcohol, como sustancia sedante que es, también produjo un aumento en esta escala. En estudios controlados en los que se administró alcohol a voluntarios sanos, la PCAG del ARCI obtuvo puntuaciones significativamente más altas que el placebo (Holdstock y de Wit, 1998; 1999) y cocaína (Foltin y cols., 1993) e incluso más altas que tras la administración de la combinación de cocaína y alcohol (Farré y cols., 1997). En un estudio más reciente, y al que ya se ha hecho referencia anteriormente, se administró alcohol y se comparó precisamente con la MDMA, la PCAG presentó puntuaciones significativamente superiores para el alcohol (Hernández-López y cols., 2002).

La escala de Somatización Ansiosa es característica de sustancias de tipo estimulante, aumentando de forma destacada para la condición de cocaína y en segundo lugar para la condición éxtasis. Este resultado está de acuerdo con numerosos estudios, experimentales y no experimentales, realizados con sustancias estimulantes como la cocaína (Preston y cols., 1992; Fischman y cols., 1976), la anfetamina (Chait y cols., 1986; Martin y cols., 1971) y la cafeína (Rush y cols., 1995; Chait, 1992). Incluso tras la administración de MDMA se observan este tipo de efectos, tanto en estudios experimentales como no experimentales (Greer y Tolbert, 1986; Dowling y 
Tabla 5. Diferencias entre cada una de las cuatro condiciones para las escalas del VESSPA

Escalas VESSPA ANOVA Test de Tukey para comparaciones múltiples

\begin{tabular}{|lccccc|}
\hline & \multicolumn{3}{c}{ Test de Tukey para comparaciones multiples } \\
\hline SEDACIÓN & $F$ & $P$ & Alcohol vs. placebo & MDMA vs. placebo & Alcohol vs. MDMA \\
\hline SOMATIZACIÓN ANSIOSA & 5.341 & 0.006 & $\uparrow^{* *}$ & N.S. & $\uparrow^{*}$ \\
\hline CAMBIOS DE PERCEPCIÓN & 19.052 & $<0,001$ & N.S. & $\downarrow^{* *}$ & N.S. \\
\hline PLACERY CONTACTO SOCIAL & 1.434 & N.S. & N.S. & $\uparrow^{* *}$ & N.S. \\
\hline ACTIVIDADY ENERGÍA & 16.123 & $<0,001$ & N.S. & $\uparrow^{* *}$ & N.S. \\
\hline SINTOMATOLOGÍA PSICÓTICA & 1.984 & N.S. & N.S. & & N.S. \\
\hline
\end{tabular}

$\uparrow:$ aumento (o $\downarrow$ disminución) del efecto del primero sobre el segundo.

${ }^{* *}: p<0,01 ;{ }^{*}: p<0,05 ;$ N.S.: No significativo.

cols., 1987; Peroutka y cols., 1988; Liester y cols., 1992; Solowij y cols., 1992; Vollenweider y cols., 1998; Tancer y Johanson, 2001), por lo que esta escala sería sensible a los mismos.

Las sustancias que producen cambios en la percepción por excelencia son las alucinógenas, en nuestro estudio representadas por la LSD. Fue esta condición la que registró una mayor puntuación en la escala de Cambios de Percepción. Estos cambios de percepción han sido descritos por consumidores recreacionales de éxtasis en entrevistas o encuestas publicadas por otros autores (McGuire y cols., 1994), sin embargo es difícil hallarlos tras la administración de MDMA en condiciones experimentales de ensayo clínico (Camí y cols., 2000; Hernández-López y cols., 2002).

La escala de Placer y Contacto Social refleja efectos típicos del éxtasis que se denominan entactógenos, sustancia para la cual presentó mayores resultados. En segundo lugar encontramos a la cocaína, algo que no sorprende conociendo los efectos euforizantes de los estimulantes (Martin y cols., 1971; Jasinski y Preston, 1986; Brauer y cols., 1995; 1996). Ya hemos puntualizado que la escala MBG del ARCI sería la indicadora de euforia y la mejor herramienta para predecir el potencial de abuso de una sustancia, así como la EAV "le gusta el fármaco" (Fischman y Foltin, 1991). La MBG aumenta en estudios controlados con cocaína (Fischman y cols., 1976; Preston y cols., 1992; Farré y cols., 1997) y MDMA (Vollenweider y cols., 1998; Camí y cols., 2000; Hernández-López y cols., 2002). También otros autores han descrito efectos similares en estudios anteriores realizando encuestas a consumidores recreacionales tras el consumo de MDMA (Downing, 1986; Greer y Tolbert, 1986), en situación simulada (Peroutka y cols., 1988; Solowij y cols., 1992; Cohen, 1995) o bien en encuestas realizadas a psicoterapeutas con experiencia en el consumo de esta sustancia (Liester y cols., 1992).
La escala de Actividad y Energía registró su mayor puntuación en la condición de cocaína. La sensación de sentirse activo, con fuerza, estar despierto, etc. es típica de las drogas de tipo estimulante como la cocaína (Fischman y cols., 1976; Preston y cols., 1992; Farré y cols., 1997) o las anfetaminas (Chait y cols., 1986; Martin y cols., 1971). El éxtasis, como sustancia estimulante que es, también aumentó esta escala considerablemente. En algunos estudios en los que se ha medido este tipo de sintomatología con otros cuestionarios, también aumentó tras el consumo de éxtasis (Davison y Parrot, 1997; Peroutka y cols., 1988; Solowij y cols., 1992; Parrot y Stuart, 1997) o de MDMA en situación experimental (Camí y cols., 2000).

Por último, y en cuanto a la escala de Sintomatología Psicótica, los síntomas de tipo psicótico son característicos de las drogas alucinógenas. De este modo, esta escala que contempla ítems de este tipo aumentó en mayor grado para la condición LSD. A lo largo de toda la literatura científica escrita sobre los efectos de la LSD existen numerosas descripciones de cuadros de tipo psicótico producidos por la misma, pudiendo provocar incluso crisis de angustia (Isbell y Logan, 1956; Isbell y cols., 1957; Claridge, 1994).

\section{Respuesta del VESSPA en un Ensayo Clínico con MDMA}

Este apartado es muy importante y quizás básico para la validación del cuestionario debido a que este es el principal contexto al que va destinado el instrumento. Como ya se ha comentado, el ensayo clínico en el que se administró el cuestionario VESSPA se dieron cuatro condiciones: MDMA, alcohol, su combinación y placebo. Los resultados obtenidos fueron en general satisfactorios como se comenta a continuación para cada una de las escalas.

La escala de sedación aumentó para la condición alcohol en comparación con placebo y MDMA. El alcohol, a diferencia de cuando se consume en lugares de 
ocio, produce efectos de tipo sedante cuando es administrado en condiciones experimentales en voluntarios sanos. Se han encontrado resultados similares con la escala PCAG del ARCI en ensayos clínicos con alcohol en comparación con placebo y también cuando se ha comparado con MDMA (Hernández-López y cols., 2002) y con otros estimulantes como la cocaína (Farré y cols., 1997; Foltin y cols., 1993).

La escala de Somatización Ansiosa mostró puntuaciones superiores tras la administración de MDMA y su combinación con alcohol respecto a placebo y alcohol. Este resultado concuerda con los resultados obtenidos por la escala LSD del $\mathrm{ARCl}$ en este mismo ensayo, reflejando ciertos efectos físicos desagradables de la sustancia (Hernández-López, 2002; Farré y cols., 2000; 2001). El efecto de tipo ansioso de la MDMA es más que conocido, llegando en numerosos casos a producir crisis de angustia en situación de ocio (Peroutka 1990; McCann y Ricaurte, 1991; 1992; Pallanti y Mazzi, 1992; McGuire y cols., 1994; Cohen, 1996).

La escala de Placer y Contacto Social registró puntuaciones superiores para la condición de MDMA. Esta escala, como ya se ha comentado, ocupa un lugar destacado en este estudio, ya que es la que describiría los efectos de tipo entactógeno del éxtasis. Recordemos que éstos no quedaban contemplados hasta el momento en otros instrumentos, por lo que su comparación con otros estudios es complicada. Ya hemos visto que en numerosas encuestas a consumidores recreacionales de éxtasis quedaban reflejados estos efectos (Peroutka y cols., 1988; Solowij y cols., 1992; Cohen, 1995) y también en estudios experimentales (Downing, 1986; Greer y Tolbert, 1986; Vollenweider y cols., 1998) donde aumentaban las puntuaciones de escalas que recogían ítems relacionados con el placer o la sensación de sentirse bien.

En la escala de Actividad y Energía se contemplaron puntuaciones significativamente más altas para la condición de MDMA. Este resultado concuerda con numerosos estudios en los que se ha demostrado que la MDMA produce efectos de estimulación tales como sensación de energía, ganas de "hacer cosas" o bien bailar cuando se está en situación de ocio (Davison y Parrot, 1997; Peroutka y cols., 1988; Solowij y cols., 1992; Parrot y Stuart, 1997). Esta es otra de las características que ha hecho popular al éxtasis.

Las escalas de Cambios de Percepción y de Sintomatología Psicótica no evidenciaron diferencias significativas en ninguna de las condiciones. El éxtasis a las dosis administradas hasta el momento en condiciones experimentales no presenta algunos de los efectos característicos cuando se consume en situación de ocio. En un estudio de Poudevida y cols. (1999) se discuten las posibles causas de estas diferencias contextuales. Por ejemplo, en cuanto a los cambios de percepción en lugares de ocio, éstos se ven influenciados por cambios de luces externas, juegos con rayos luminosos, cambios de intensidad lumínica, exposición de imágenes virtuales, etc. En referencia a Sintomatología Psicótica, aunque se ha descrito en situaciones de ocio (Schifano, 1991; Creighton y cols., 1991; Huckle y cols., 1991; Series y cols., 1994), es raro que se de en situación experimental (Hermle y cols., 1993). Ha de tenerse en cuenta que un ensayo clínico cuenta con unas normas éticas y de seguridad que salvaguardan en la medida de lo posible la salud del sujeto y el impacto negativo de la sustancia sobre el mismo. Por lo tanto las dosis administradas eran quizá demasiado bajas para producir cambios de percepción o cuadros de tipo psicótico (dosis que aumentan cuando la sustancia es autoadministrada por el sujeto en situación de ocio).

En conclusión, puede afirmarse que se ha construido y validado un cuestionario sensible a los efectos subjetivos del éxtasis o MDMA (para la cual no existían cuestionarios estandarizados y validados hasta el momento) y de otras sustancias con potencial de abuso como la cocaína, la LSD, el cannabis y el alcohol. Además, el cuestionario VESSPA ha mostrado ser un instrumento válido cuando es administrado tanto en una situación simulada como en una situación experimental de ensayo clínico.

Este proyecto ha sido financiado en parte por la Generalitat de Catalunya (Soporte a los grupos de investigación consolidados en Catalunya, CIRIT 1999SGR00242, 2001SGR00407). Agradecemos a Jordi Pérez (UPF) su ayuda en la validación del cuestionario.

Este proyecto ha sido el ganador del premio annual Zambón-Socidrogalcohol 2003.

Aquellas personas interesadas en el cuestionario VESSPA pueden solicitar una copia del mismo al autor de correspondencia.

\section{BIBLIOGRAFÍA}

Adams, G.S. (1975). Medición y evaluación. Barcelona: Herder.

Anastasi, A. (1982). Tests psicológicos (3 $3^{\text {a }}$ edición). Madrid: Aguilar.

Arasteh, K., Poudevida, S., Farré, M., Roset, P.N., Camí, J. (1999). Response patterns of the Spanish version of the 49-item short form of the Addiction Reserch Center Inventory (ARCl) after the use of sedatives, stimulants, and opioids. Drug and Alcohol Dependence, 55, 117125.

Brauer, L.H., Ambre, J. \& de Wit, H. (1996). Acute tolerance to subjective but not cardiovascular effects of d-amphetamine in normal, healthy men. Journal of Clinical Psychopharmacology, 16, 72-76. 
Brauer, L.H., Rukstails, M.R. \& de Wit, H. (1995). Acute subjective responses to paroxetine in normal volunteers. Drug and alcohol Dependence, 39, 223-230.

Burnat, P., Le Brumant-Payen, C., Huart, B., Ceppa, F. \& PaiIler, F.M. (1996). L'ecstasy: psychostimulant, hallucinogène et toxique. La Presse Médicale, 25, 1208-1212.

Camí, J. y Farré, M. (1996). Éxtasis, la droga de la ruta del bakalao. Medicina Clínica (Barcelona), 106, 711-716.

Camí, J., Farré, M., Mas, M., Roset, P.N., Poudevida, S., Mas, A., San, L. \& de la Torre, R. (2000). Human Pharmacology of 3,4-Methylenedioxy-methamphetamine ("Ecstasy"): Psychomotor Performance and Subjective Effects. Journal of Clinical Psichopharmacology, 20, 455-466.

Claridge, G. (1994). LSD: A missed opportunity. Human Psychopharmacology, 9, 343-351.

Cohen, R.S. (1995). Subjective reports on the effects of the MDMA (ecstasy) experience in humans. Progress in Neuropsychopharmcology and Biological Psychiatry, 19, 1137-1145

Cohen, R.S. (1996). Adverse symptomatology and suicide associated with the use of methylenedioxymethamphetamine (MDMA, ecstasy). Biological Psychiatry, 39, 819-820.

Cone, E.J. \& Johnson, R.E. (1988b). Marijuana-laced brownies: Bejavioral effects, physiologic effects, and urinalysis in humans following ingestion. Journal of Analytical Toxicology, 12, 169-175.

Cone, E.J., Welch, P. \& Lange, W.R. (1988a). Clonidine partially blocks the physiologic effects but not the subjective effects produced by smoking marijuana in male human subjects. Pharmacology Biochemistry and Behaviour, 29, 649-652.

Creighton, F.J., Black, D.L. \& Hyde, C.E. (1991). Ecstasy psychosis and flashbacks. British Journal of Psychiatry, 159, 713-715.

Curran, H.V. \& Travill, R.A. (1997). Mood and cognitive effects of \pm 3 ,4-methylenedioxymethamphetamine (MDMA, 'ecstasy'): week-end 'high' followed by midweek low. Addiction, 92, 821-831.

Chait, L.D. (1992). Factors influencing the subjective response to caffeine. Behavioural Pharmacology, 3, 219.

Chait, L.D. (1993). Factors influencing the reinforcing and subjective effects of d-amphetamine in humans. Behavioural Pharmacology, 4, 191-199.

Chait, L.D., Evans, S.M., Grant, K.A., Kamien, J.B., Johanson, C.E. \& Schuster, C.R. (1988). The discriminative stimulus and subjective effects of smoked marijuana in humans. Psychopharmacology, 94, 206-212.

Chait, L.D., Uhlenhuth, E.H. \& Johanson, C.E. (1986). The discriminative stimulus and subjective effects of phenylpropanolamine, mazindol and d-amphetamine in humans. Pharmacology Biochemistry and Behavior, 24, 1665-1672.

Davison, D. \& Parrott, C. (1997). Ecstasy (MDMA) in recreational users: self-reported psychological and physiological effects. Human Psychopharmacology, 12, 221-226. de la Fuente, L., Rodríguez, M.A., Orta, J.V., Sánchez, J. Y Barrio G. (1997). Epidemiología del consumo de drogas de diseño en España. Medicina Clínica (Barcelona), 108, 54-61.

Delegación del Gobierno para el Plan Nacional sobre Drogas (1996, 1997, 1998, 1999, 2000 y 2001). Observatorio Español sobre Drogas. Informes n¹, 2, 3 y 4. Madrid: Ministerio del Interior.

Dowling, G.P., McDonough, E.T. \& Bost, R.O. (1987). 'Eve' and 'Ecstasy". A report of five deaths associated with the use of MDEA and MDMA. JAMA, 257, 1615-1617.

Downing, J. (1986). The psychological and physiological effects of MDMA on normal volunteers. Journal of Psychoactive Drugs, 18, 335-340.

Farré, M., de la Torre, R., González, M.L., Terán, M.T., Roset, P.N., Menoyo, E. \& Camí, J. (1997). Cocaine and alcohol interactions in humans: neuroendocrine effects and cocaethylene metabolism. Journal of Pharmacology and Experimental Therapeutics, 283, 164-176.

Farré, M., Roset, P.N., Hernández-López, C., Poudevida, S., Menoyo, E., de la Torre, R., Ortuño, J., Peiró, A. \& Camí, J. (2001). Repeated adminstration of MDMA to healthy volunteers. Drug and Alcohol Dependence, 63, (Suppl. 1), S46.

Farré, M., Roset, P.N., Tomillero, A., Hernández-López, C., Poudevida, S., Menoyo, E., Sánchez, I., de la Torre, R., Ortuño, J. \& Camí, J. (2000). Repeated administration of ecstasy to humans: preliminary findings. British Journal of Clinical Pharmacology, (Suppl. 49), 427.

Farré, M., Terán, M.T., \& Camí, J. (1996). A comparison of the acute behavioral effects of flunitrazepam and triazolam in healthy volunteers. Psychopharmacology, 125, $1-12$.

Ferrando, J. y Pérez J. (1996). Un instrumento para medir quemazón profesional en los docentes: adaptación catalana del "Maslach Burnout Inventory (MBI)". Revista de Psiquiatria de la Facultad de Medicina de Barcelona, 23, 11-18.

Fischman, M.W. \& Foltin, R.W. (1991). Utility of subjectiveeffects measurements in assessing abuse liability of drugs in humans. General methods of drug abuse liability assessment. British Journal of Addiction, 86, 1563-1570.

Fischman, M.W., Schuster, C.R., Resnekov, L., Shick, J.F.E., Krassnegor, N.A., Fennell, W. \& Freedman, D.X. (1976). Cardiovascular and subjective effects of intravenous cocaine administration in humans. Archives of General Psychiatry, 33, 983-989.

Foltin, R.W., Fischman M.W., Pippen, P.A. \& Kelly, T.H. (1993). Behavioral effects of cocaine alone and in combination with ethanol or marijuana in humans. Drug and Alcohol Dependence, 32, 93-106.

Greer, G., \& Tolbert, R. (1986). Subjective reports of the effects of MDMA in a Clinical Setting. Journal of Psychoactive Drugs, 18, 319-327.

Grob, C.S., Poland, R.E., Chang, L. \& Ernst, T. (1996). Psychobiologic effects of 3,4-methylenedioxymethamphetamine in humans: methodological considerations and 
preliminary observations. Behavioural Brain Research, 73, 103-107.

Haertzen, C.A. (1966). Development of scales based on patterns of drug effects, using the Addiction Research Center Inventory (ARCI). Psychological Reports, 18, 163-194.

Henry, J.A., Jefreys, K.J. y Dawling, S. (1993). Intoxicación y muerte por 3,4-metilendioximetanfetamina (éxtasis). The Lancet (ed. española), 22, 3-7.

Hermle, L., Spitzer, M., Borchardt, D., Kovar, KA. \& Gouzoulis, E. (1993). Psychological effects of MDE in normal subjects. Are entactogens a new class of psychoactive agents?. Neuropsychopharmacology, 8, 171-176.

Hernández-López, C., Farré, M., Roset, PN., Menoyo, E., Pizarro, N., Ortuño, J., Torrens, M., Camí, J. \& de la Torre, R. (2002). 3,4-Methylenedioxymethamphetamine (MDMA, Ecstasy) and Alcohol Interactions in Humans: Psychomotor Performance, Subjective Effects and Pharmacokinetics. Journal of Pharmacology and Experimental Therapeutics, 300, 236-244.

Higgins, S.T. \& Stitzer, M.L. (1986). Acute marijuana effects on social conversation. Psychopharmacology, 89, 234-238.

Holdstock, L. \& de Wit, H. (1998). Individual differences in the biphasic effects of ethanol. Alcoholism, Clinical and Experimental Research, 22, 1903-1911.

Holdstock, L. \& de Wit, H. (1999). Individual differences in subjective responses to ethanol and triazolam. Behavioral Pharmacology, 10, 283-295.

Holzemer, W.L, Henry, S.B., Nokes, K.M., Corless, I.B., Brown, M.A., Powell-Cope, G.M., Turner, J.G. \& Inouye, J. (1999). Validation of the sign and symptom chek-list for persons with HIV disease (SSC-HIV). Journal of advanced nursing, 30, 1041-1049.

Huckle, P., Palmer, D., Lester, H. \& Huws, D. (1991). Case report: Ecstasy. The road to psychosis?. British Journal of Clinical Psychiatry, 8, 4-5.

Isbell, H. \& Logan, C.R. (1956). Studies on the Diethylamide of Lysergic Acid (LSD-25): II. Effects of chlorpromazine, azacyclonol, and reserpine on the intensity of the LSDreactions. Archives of Neurology and Psychiatry, 77, 350-358.

Isbell, H., Logan, C.R. \& Miner, E.J. (1957). Studies on the Diethylamide of Lysergic Acid (LSD-25): III. Attempts to attenuate the LSD-reaction in man by preteatment with neurohormanal blocking agents. Archives of Neurology and Psychiatry, 81, 20-27.

Jasinski, D. \& Preston, K.L. (1986). Evaluation of mixtures of morphine and d-amphetamine for subjective and physiological effects. Drug and Alcohol Dependence, 17, $1-13$.

Johanson, C.E. (1991). Discriminative stimulus effects of diazepam in humans. Journal of the Experimental Analysis of Behavior, 257, 634-643.

Lamas, X., Farré, M., Llorente, M. \& Camí, J. (1994). Spanish version of the 49-item short form of the Addiction Research Center Inventory (ARCI). Drug and Alcohol Dependence, 35, 203-209.
Liester, M.B., Grob, C.S., Bravo, G.L. \& Walsh, R.N. (1992). Phenomenology and sequelae of 3,4-Methylenedioxymethamphetamina use. Journal of Nervous and Mental Disease 180, 345-352.

Martin, W.R., Sloan, J.W., Sapira, J.D. \& Jasinski, D.R. (1971). Physiologic, subjective, and behavioral effects of amphetamine, methamphetamine, ephedrine, phenmetrazine, and methylphenidate in man. Clinical Pharmacology and Therapeutics, 12, 245-258.

McCann, U. \& Ricaurte, G.A. (1991). Lasting neuropsychiatric sequelae of metylenedioximethamphetamine (Ecstasy) in recreational users. Journal of Clinical Psychopharmacology, 11, 302-305.

McCann, U. \& Ricaurte, G.A. (1992). MDMA (Ecstasy) and panic disorder. Biological Psychiatry, 32, 950-953.

McGuire, P., Cope, H. \& Fahy, T. (1994). Diversity of psychopathology associated with use of 3,4-methylenedioxymethamphetamine ("ecstasy"). British Journal of Psychiatry, 165, 391-395.

Nemeth-Coslett, R., Henningfield, J.E., O'Keeffe, M.K. \& Giffiths, R.R. (1986). Effects of marijuana smoking on subjective ratings and tobacco smoking. Pharmacology Biochemistry and Behavior, 29, 649-652.

Nunnally, J.C. \& Bernstein, I.H. (1994). Psychometric Theory (3rd edition). New York: McGraw-Hill.

Observatorio Europeo de las Drogas y las Toxicomanías (2000). Informe anual sobre el problema de la drogodependencia en la Unión Europea. Lisboa.

Pallanti, S. \& Mazzi, D. (1992). MDMA (Ecstasy) precipitation of panic disorder. Biological Psychiatry, 32, 91-95.

Parrot, A.C. \& Stuart, M. (1997). Ecstasy (MDMA), amphetamine, and LSD: Comparative mood profiles in recreational polydrug users. Human Psychopharmacology, $12,501-504$.

Pérez, J. y Torrubia, R. (1986). Fiabilidad y validez de la versión española de la Escala de Búsqueda de Sensaciones (forma V). Revista Latinoamericana de Psicología, 18, 7-22.

Peroutka, S.J. (1990). Ecstasy: the clinical, pharmacological and neurotoxicological effects of the drug MDMA. Dordrecht: Kluwer.

Peroutka, S.J., Newman, H. \& Harris, H. (1988). Subjective effects of 3,4-Methylene-dioxymethamphetamina in recreational Users. Neuropsychopharmacology, 1, 273-277.

Poudevida, S., Farré, M., Mas, M. y Camí, J. (1999). Objetivar lo subjetivo, de la fiesta al laboratorio. Ulises, 2, 3338.

Poudevida, S., Farré, M., Mas, M., Roset, P.N. y Camí, J. (1997). Elaboración de un instrumento para evaluar los efectos subjetivos producidos por el éxtasis en condiciones experimentales. IV Congreso de la Sociedad Española de Toxicomanías, Santander. Libro de resúmenes, 117.

Preston K.L., Sullivan J.T., Strain, E.C. \& Bigelow, G.E. (1992). Effects of cocaine alone and in combination with bromocriptine in human cocaine abusers. Journal 
of Pharmacology and Experimental Therapeutics, 262, 279-291.

Renom, J., Solanas, A. y Puyuelo, M. (1995). Análisis psicométrico de una prueba de lenguaje y su adaptación informática. Revista de logopedia, foniatría y audiología, 15, 121-137.

Riba, J., Rodríguez-Fornells, A., Urbano, G, Morte, A., Antonijoan, R., Montero, M., Callaway, J.C. \& Barbanoj, M.J. (2001a). Subjective effects and tolerability of the South American psichoactive beverage Ayahuasca in healthy volunteers. Psychopharmacology, 154, 85-95.

Riba, J., Rodríguez-Fornells, A., Strassman, R.J. \& Barbanoj, M.J. (2001b). Psychometric assessment of the Hallucinogen Rating Scale. Drug and Alcohol Dependence, 62, 215-223.

Rush, C.R., Sullivan, J.T. \& Griffiths, R.R. (1995). Intravenous caffeine in stimulant drug abusers: Subjective reports and physiological effects. Journal of Pharmacology and Experimental Therapeutics, 273, 351-358.

Schifano, F. (1991) Chronic atypical psychosis associated with MDMA ("ecstasy") abuse. The Lancet, 338, 1335.

Series, H., Boeles, S., Dorkins, E. \& Peveler, R. (1994). Psychiatric complications of ecstasy use. Journal of Psychopharmacology, 8, 60-61.

Smith, L.F. (1999). The WOMB (Women's views of birth) antenatal satisfaction questionnaire: development, dimensions, internal reliability, and validity. British journal of general practice, 49, 971-975.

Solowij, N., Hall, W. \& Lee, N. (1992). Recreational MDMA use in Sydney: a profile of 'Ecstasy' users and their experiences with the drug. British Journal of Addiction, 87, 1161-1172.
Strassman, R.J., Qualls, C.R., Uhlenhuth, E.H. \& Kellner, R. (1994). Dose-response study of N,N-dimethyltriptamine in humans. II. Subjective effects and preliminary results of a new rating scale. Archives of General Psychiatry, 51, 98-108.

Tancer, M.E. \& Johanson C.E. (2001). The subjective effects of MDMA and mCPP in moderate MDMA users. Drug and Alcohol Dependence, 65, 97-101.

Tange, C. (1999). Construction of a scale of attitude toward death in adolescence and its validity and reliability. Shinrigaku Kenkyu, 70, 327-332.

Terán, M.T., Farré, M., Lamas, X., Ugena, B. \& Camí, J. (1993). Subjective and psychomotor effects of flunitrazepam in healthy volunteers. In: L. Harris (Ed.), Problems of Drug Dependence. NIDA Research Monograph, 132, 348.

Vollenweider, F.X., Gamma, A., Liechti, M. \& Huber, T. (1998). Psychological and cardiovascular effects and short-term sequelae of MDMA ("Ecstasy") in MDMA-naïve healthy volunteers. Neuropsychopharmacology, 19, 241-251.

Warot, D., Danjau, P., Payan, C. \& Puech, A.J. (1997). Sensitivity and specificity to amphetamine of a French version of the 49-items short form of the Addiction Research Center Inventory. Drug and Alcohol Dependence, 45, 177-183.

Williamson, S., Gossop, M., Powis, B., Griffiths, P., Fountain, J. \& Strang, J (1997). Adverse effects of stimulant drugs in a community sample of drug users. Drug and Alcohol Dependence, 44, 87-94.

World Medical Association (1996). Declaration of Helsinki. South Africa: Somerset West. 